\title{
Medication-related inpatient falls: a critical review
}

\author{
Tatiane Bomfim Ribeiro*1, Daniela Oliveira de Melo², Flávia de Oliveira Motta Maia ${ }^{3}$, \\ Eliane Ribeiro ${ }^{4}$
}

\begin{abstract}
${ }^{1}$ Hospital Universitário da Universidade de São Paulo, Departamento de Farmácia, São Paulo, SP, Brasil, ${ }^{2}$ Universidade Federal de São Paulo, Departamento de Fisiologia e Farmacologia, Diadema, SP, Brasil, ${ }^{3}$ Hospital Universitário da Universidade de São Paulo, Departamento de Enfermagem, São Paulo, SP, Brasil, ${ }^{4}$ Hospital Universitário da Universidade de São Paulo, Departamento de Farmácia, São Paulo, SP, Brasil e Universidade de São Paulo, Faculdade de Ciências Farmacêuticas, Departamento de Farmácia, São Paulo, SP, Brasil
\end{abstract}

\begin{abstract}
Falls are the second leading cause of accidental and unintentional injury deaths worldwide. Inpatient falls in hospital settings are likely to prolong the length of stay of patients in nearly 6.3 days, leading to increased hospitalization costs. The causes of fall incidents in healthcare facilities are multifactorial in nature and certain medications use could be associated with these incidents. This review seeks to critically evaluate the available literature regarding the relationship between inpatient falls and medication use. A comprehensive search was performed on MEDLINE, EMBASE and Lilacs with no time restriction. The search was filtered using English, Spanish or Portuguese languages. Our study evaluated medication use and inpatients falls that effectively happen, considering all ages and populations. An assessment of bias and quality of the studies was carried out using an adapted tool from the literature. The drugs were classified according to the Anatomic Therapeutics Chemical Code. The search strategy retrieved 563 records, among which 23 met the eligibility criteria; ninety three different pharmacological subgroups were associated with fall incidents. Our critical review suggests that the use of central nervous system drugs (including anxiolytics; hypnotics and sedatives; antipsychotics; opioids; antiepileptics and antidepressants) has a greater likelihood of causing inpatient falls. A weak relationship was found between other pharmacological subgroups, such as diuretics, cardiovascular system-related medications, and inpatient fall. Remarkably, several problems of quality were encountered with regard to the eligible studies. Among such quality problems included retrospective design, the grouping of more than one medication in the same statistical analysis, limited external validity, problems related to medication classifications and description of potential confounders.
\end{abstract}

Keywords: Accidental Fall/hospitals. Therapeutic Uses. Inpatients. Central Nervous System Agents.

\section{INTRODUCTION}

Falls are the second leading cause of accidental and unintentional injury deaths in Brazil and worldwide (CDC, 2014; DataSUS, 2014; WHO, 2004). In healthcare facilities, this adverse event is a prevalent patient safety problem. The reduction of injuries stemming from fall incidents is one of the six main goals of the Worldwide Alliance for Patient Safety (WHO, 2008). The World Health Organization (WHO) defines fall as "inadvertently coming to rest on the ground, floor, or other lower level, excluding intentional change in position" (WHO, 2007).

\footnotetext{
*Correspondence: T. B. Ribeiro. Hospital Universitário, Universidade de São Paulo. Av. Prof. Lineu Prestes, 2565, 05508-000, Butantã, São Paulo - SP. E-mail: tatianeribeiro6@gmail.com
}

As of 2012, the fall rate of adult inpatients in the USA was 3.56 per 1,000 patients/day (pd), out of which $26.1 \%$ resulted in injury (Bouldin et al., 2012). Inpatient falls in hospital settings are likely to prolong the length of stay of patients in nearly 6.3 days, leading to increased direct and indirect hospitalization costs of $\$ 13,316$ in three American hospitals (Wong et al., 2011). There are few data on adult inpatient falls in Brazil, and most of the studies conducted on the issue restricted their focus to elderly patients. In a study carried out in three large general hospitals in a single state in Brazil, the rate of fall among elderly inpatients was 12.6 per 1,000 patients a day (Abreu et al., 2015).

The fall risk factors are multifactorial in nature and are conventionally classified into intrinsic and extrinsic factors (Ferreira, Yoshitome, 2010, Severo et al., 2014). 
The intrinsic factors are associated with the individual and are essentially related to factors such as the illness of the patient, elderly physiological changes, cognitive decline, gait alteration and medication use (Buksman et al., 2008). These factors are found to be transitory, and include orthostatic hypotension, syncope after anesthesia (Spoelstra et al., 2012) or polypharmacy (Richardson, Bennett, Kenny, 2014). The extrinsic factors are related to external conditions including environmental hazards (such as bedrails or footwear) and low levels of nursing care (Severo et al., 2014; Hignett, Masud, 2006).

While current research studies have devoted their attention to understanding the causes of inpatient fall risks, the huge challenge lies in devising efficient strategies for the prevention of falls in healthcare facilities (Miake-Lye et al., 2013; Brasil, 2013b; Rezende, Gaede-Carrillo, Sabastião, 2012, Boushon et al., 2012). Medication use is cited as one of the main causes of inpatient falls (Rubenstein, 2006; Boyle, Naganathan, Cumming, 2010). Some studies point out the need for the revision of inpatient medical prescriptions and the inclusion of a clinical pharmacist into the multiprofessional team so as to increase patient safety and reduce the risk of fall (Zermansky et al., 2006; Haumschild et al., 2003; Browne, Kingston, Keane, 2014). Most of the drugs classified as high risk for fall are categorized in this manner as a result of the adverse events that these medications can cause. Among these adverse effects include orthostatic hypotension due to antihypertensives use or hypoglycemia by virtue of the use of antidiabetics (Rubenstein, 2006; Boushon et al., 2012).

Most of the reviews published in the literature on this subject sough to analyze the underlying relationship between medication use and fall incidents in different settings including community settings, long-term facilities and hospitals. The sample population investigated was often restricted to elderly patients and the studies mostly considered only few medication subgroups (Park et al., 2015; Zang, 2013; Bloch et al., 2010; Boyle, Naganathan, Cumming, 2010; Woolcott et al., 2009; Hegeman et al.,
2009; Campbell, 1991). To date, no reports have been published in the literature involving specific medications related to inpatient falls without age restriction. Clearly, in a hospital setting, patients are exposed to a wide range of medications and specific conditions that may contribute towards altering the risk of falling (Matarese et al., 2014). The aim of this study is to carry out a thorough assessment of the literature available regarding the relationship between medication and inpatient falls, regardless of the age of patients. The paper will also undertake a critical review in this context.

\section{METHODS}

\section{Data sources and search}

A comprehensive literature review was conducted by the author, T.B.R, on MEDLINE (by Pubmed), EMBASE (by Elsevier), and Lilacs (by Virtual Health Library website) (last search on August 4, 2016), with no time restriction and filtered by English, Spanish or Portuguese languages. The medical subject heading (MESH) terms used included "inpatient", "accidental fall", and "therapeutic uses" (linked with "AND"). The term "therapeutic uses" was chosen by virtue of the fact that it indexed all medication classes. A similar search was done on EMBASE using the Emtree terms "hospital", "falling" and "drug therapy". All terms were expanded to capture all relevant articles using relevant synonyms including hospital*, drug*, medicat* and fall*. All potentially eligible studies were included with the exception of reviews. The search strategy is presented in Table I. Some bibliographical references in the eligible studies were evaluated. In some cases, these references were either withdrawn or included based on our search strategy.

\section{Study selection}

Studies were considered eligible for inclusion once

TABLE I - Search strategy

\begin{tabular}{ll}
\hline Database & \multicolumn{1}{c}{ Search Strategy } \\
\hline PUBMED.MEDLINE & $((((($ inpatients[MeSH Terms]) OR inpatient*[Title]) OR hospital*[Title])) AND (((therapeutic \\
& $\begin{array}{l}\text { uses[MeSH Terms]) OR drug*[Title]) OR medicat*[Title])) AND ((accidental falls[MeSH Terms]) } \\
\text { OR fall*[Title]) }\end{array}$ \\
\hline EMBASE & $\begin{array}{l}\text { inpatient*:ti OR 'hospital patient':de,ti OR hospital:ti AND (drug*:ti OR 'drug therapy'/exp OR } \\
\text { medicat*:de,ti) AND ('fall risk':de OR falling:de OR fall*:ti) }\end{array}$ \\
\hline BVS.LILACS & $\begin{array}{l}\text { (mh:inpatients OR ti:inpatient* OR ti:hospital*) AND (mh:”therapeutic uses" OR ti:drug* OR } \\
\text { ti:medicat* OR mh:drug* OR mh:medicat*) AND (mh:”accidental falls" OR ti:fall*) }\end{array}$ \\
\hline
\end{tabular}


they contained original data based on the association between medication use and inpatient falls, were exclusively conducted in a hospital setting, and included all ages and special populations. The author thoroughly read and assessed all titles and abstracts and selected the studies of interest in line with the inclusion criteria. Reviews and studies that considered pre-hospitalization falls, facilities different from hospital (community or long-term care facilities), and those that did not evaluate medication or falls that effectively happen in a hospital setting were all excluded. All studies retrieved in the search were included in our investigation without regard to quality limitations. The excluded studies were not categorized.

\section{Data extraction and quality assessment}

Data were extracted and summarized in a spreadsheet with the main study information including the following: author/year, country, number of fallers/ number of control group and special sample condition, median age of fallers, follow-up period, other factors related to fall, medication and measure of association. The type of the studies design (cohort, case-control, etc.) was classified by the authors.

Medications that were related to inpatient fall, those ones which were statistically significant according to univariate and/or multivariate analysis, were classified based on the Anatomical Therapeutic Chemical (ATC) classification. These medications were graded in the pharmacological groups ( $3^{\text {rd }}$ level). It is worth noting that those medications that could not be classified as $3^{\text {rd }}$ level (due to the absence of author specification) were catalogued according to the therapeutic subgroup $\left(2^{\text {nd }}\right.$ level).

Data were extracted on the most cited pharmacological subgroups and details were provided regarding the studies that mentioned these subgroups. The data details included the special populations and medications involved.

All studies were evaluated using a quality tool adapted from von Elm (2007) and Young and Solomon (2009) proposals on "how to critically appraise an article". This tool evaluates the main factors that can induce study bias. Among such factors include the following: clear population definition and representation; selection of control and case groups (criteria and sample); full identification of potential confounders; accurate outcome measures; confounders and relevant exposure; and adequate statistical analysis (Table III). These factors were classified under the following categories: "high risk of bias", "low risk of bias" and "not clear". This classification was based on the completion of the topics about the assessment of the risk of bias included in Table III.

\section{RESULTS}

The search strategy retrieved 563 records, 34 of which were considered for full-text screening while 23 met the eligibility criteria (Figure 1).

A summarized presentation of the studies data can be found in Table III. Most of the studies (87\%) included were published after 2004. Sixty one percent (14/23)

TABLE II - Bias Analysis Tool adapted from Strobe (2007) and Young and Solomon (2009) proposals on "how to critically appraise an article",

Clear definition and representation of population

Adequate selection of cases and control (or comparative group)

Full identification of potential confounders

Accurate measure of all important relevant exposure, potential confounders and outcomes
-The population, the eligibility criteria and methods of selection were clearly described; -The sample was representative of the population or group studied

-The case and control(or comparative group) selection criteria were clearly described;

-The control (in case it is control case) reflects the case population (characteristics and number);

- The same indicators were evaluated for both groups.

- All confounders (or at least 80\%) were identified (we considered the main confounders to fall: history of fall, age, ambulatory aid, gait, mental status (dementia, confusion, disorientation, impulsivity), symptomatic depression), according to Hendrich II Fall Risk Model and Morse Fall Scale.

-Accurate measure and description of the relevant exposure, potential confounders and outcomes, sample characteristics, follow-up period and clear outcomes.

-Description and accurate analysis of the statistical methods chosen. 
TABLE III - Eligible studies data

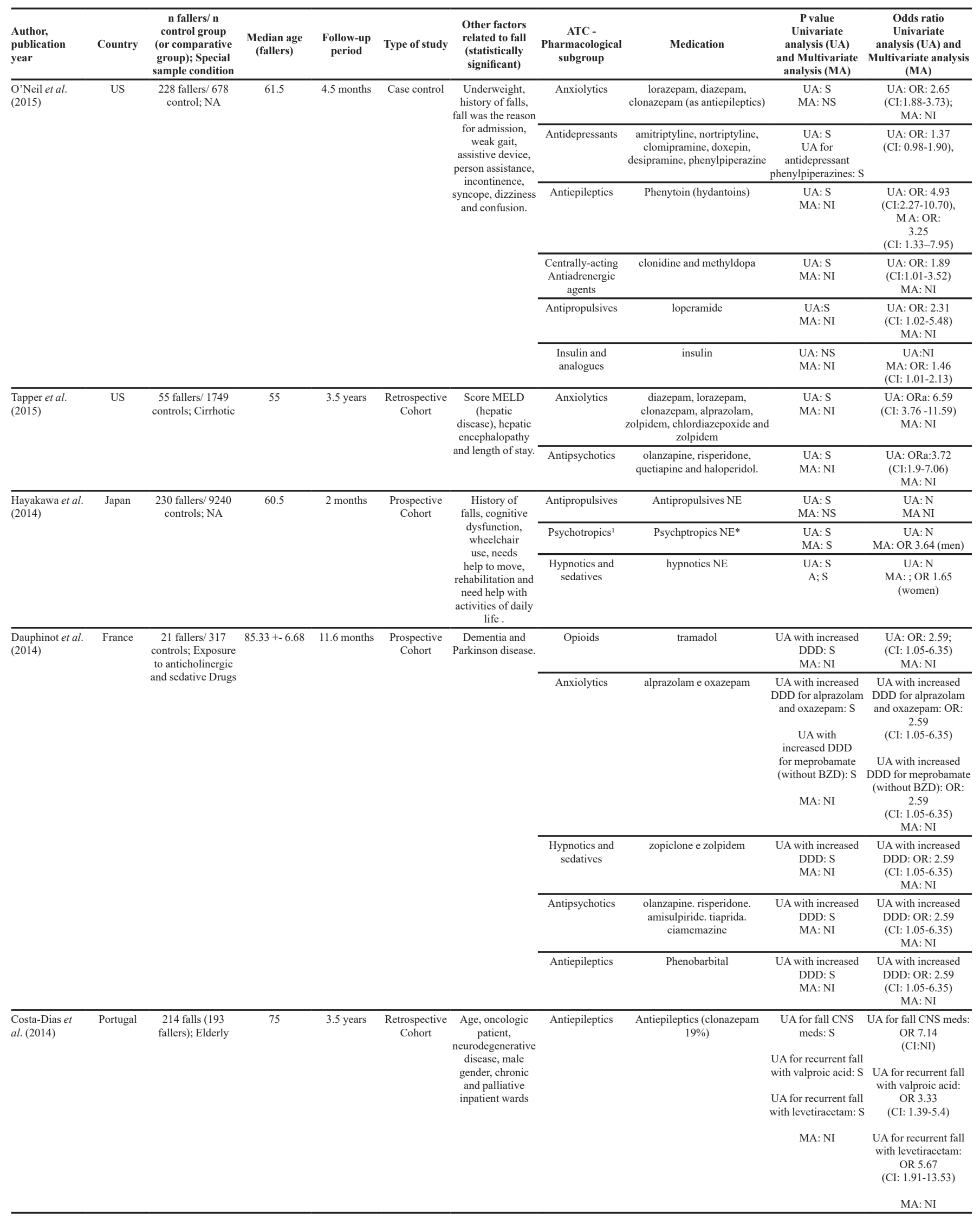


TABLE III - Eligible studies data (cont.)

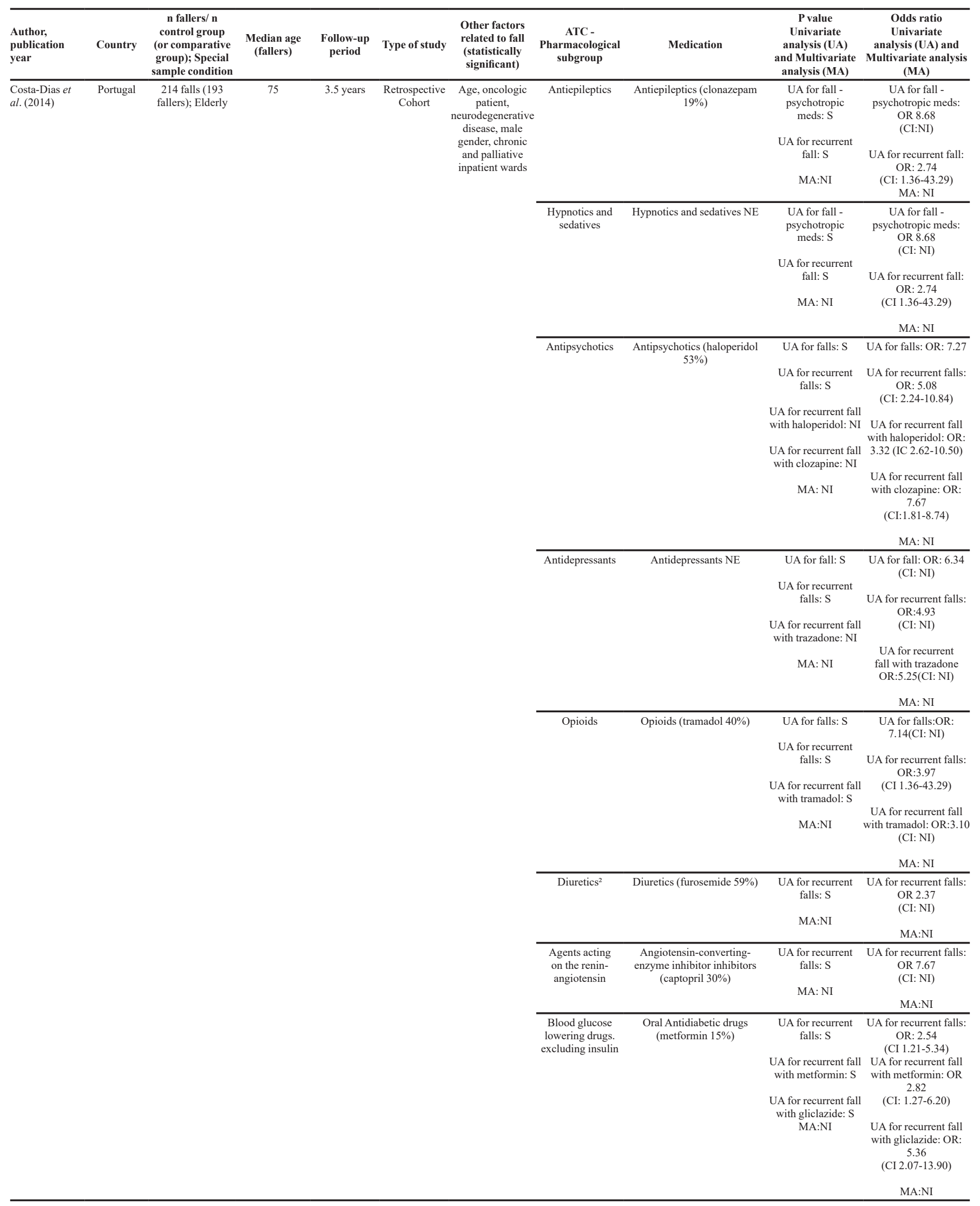


TABLE III - Eligible studies data (cont.)

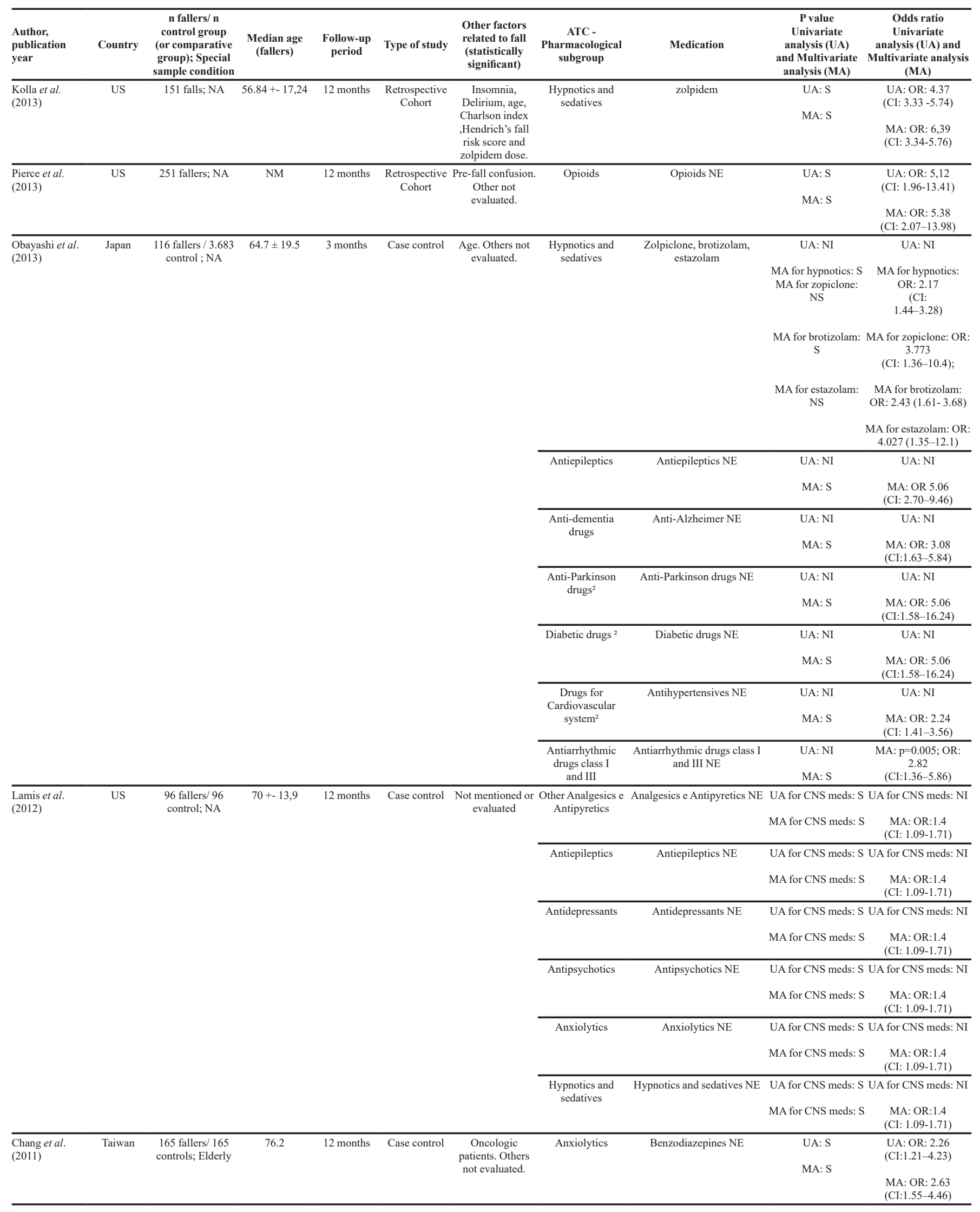


TABLE III - Eligible studies data (cont.)

\begin{tabular}{|c|c|c|c|c|c|c|c|c|c|c|}
\hline $\begin{array}{l}\text { Author, } \\
\text { publication } \\
\text { year }\end{array}$ & Country & $\begin{array}{c}\mathbf{n} \text { fallers/ } \mathbf{n} \\
\text { control group } \\
\text { (or comparative } \\
\text { group); Special } \\
\text { sample condition } \\
\end{array}$ & $\begin{array}{l}\text { Median age } \\
\text { (fallers) }\end{array}$ & $\begin{array}{c}\text { Follow-up } \\
\text { period }\end{array}$ & Type of study & $\begin{array}{c}\text { Other factors } \\
\text { related to fall } \\
\text { (statistically } \\
\text { significant) }\end{array}$ & $\begin{array}{c}\text { ATC - } \\
\text { Pharmacological } \\
\text { subgroup }\end{array}$ & Medication & $\begin{array}{c}\text { Pvalue } \\
\text { Univariate } \\
\text { analysis (UA) } \\
\text { and Multivariate } \\
\text { analysis (MA) } \\
\end{array}$ & $\begin{array}{c}\text { Odds ratio } \\
\text { Univariate } \\
\text { analysis (UA) and } \\
\text { Multivariate analysi } \\
\text { (MA) } \\
\end{array}$ \\
\hline \multirow[t]{6}{*}{$\begin{array}{l}\text { Chang et al. } \\
\text { (2011) }\end{array}$} & \multirow[t]{6}{*}{ Taiwan } & \multirow[t]{6}{*}{$\begin{array}{l}165 \text { fallers/ } 165 \\
\text { controls; Elderly }\end{array}$} & \multirow[t]{6}{*}{76.2} & \multirow[t]{6}{*}{12 months } & \multirow[t]{6}{*}{ Case control } & \multirow{6}{*}{$\begin{array}{c}\text { Oncologic } \\
\text { patients. Others } \\
\text { not evaluated. }\end{array}$} & Opioids & \multirow[t]{2}{*}{ Opioids NE } & UA: NI & UA: NI \\
\hline & & & & & & & & & MA: S & $\begin{array}{l}\text { MA: OR: } 2.13 \\
\text { (CI:1.16-3.94) }\end{array}$ \\
\hline & & & & & & & \multirow[t]{2}{*}{$\begin{array}{l}\text { Antihistamines for } \\
\text { systemic use }\end{array}$} & \multirow[t]{2}{*}{ Antihistamines NE } & UA: NI & UA: NI \\
\hline & & & & & & & & & MA: S & $\begin{array}{l}\text { MA: OR: } 3.00 \\
\text { (CI:1.19-7.56) }\end{array}$ \\
\hline & & & & & & & \multirow[t]{2}{*}{$\begin{array}{l}\text { Hypnotics and } \\
\text { sedatives }\end{array}$} & \multirow[t]{2}{*}{ zolpidem } & UA: NI & UA: NI \\
\hline & & & & & & & & & MA: S & $\begin{array}{l}\text { MA: OR: } 2.38 \\
\text { (CI: } 1.04-5.43)\end{array}$ \\
\hline $\begin{array}{l}\text { Cashin, Yang } \\
\text { (2011) }\end{array}$ & Canada & 151 falls; NA & 73.5 & 12 months & $\begin{array}{c}\text { Cross sectional } \\
\text { study }\end{array}$ & $\begin{array}{l}\text { Not mentioned or } \\
\text { evaluated }\end{array}$ & Anxiolytics & $\begin{array}{l}\text { Alprazolam, bromazepam, } \\
\text { chlordiazepoxide, clobazam. } \\
\text { Clonazepam, clorazepate. } \\
\text { Diazepam,flurazepam. } \\
\text { Lorazepam,midazolam, } \\
\text { nitrazepam,oxazepam, } \\
\text { temazepam,triazolam }\end{array}$ & $\begin{array}{l}\text { UA: NI } \\
\text { MA: NI }\end{array}$ & $\begin{array}{l}\text { UA: NI } \\
\text { MA: NI }\end{array}$ \\
\hline $\begin{array}{l}\text { Bun, Serby, } \\
\text { Friedmann } \\
\text { (2011) }\end{array}$ & US & $\begin{array}{c}15 \text { fallers / } 233 \\
\text { control; Psychiatric } \\
\text { population with } \\
\text { hyponatemia }\end{array}$ & 55,8 & 12 months & Case control & $\begin{array}{c}\text { Age and } \\
\text { hyponatermia. } \\
\text { Others not } \\
\text { evaluated. }\end{array}$ & Antipsychotics & Antipsychotics NE & $\begin{array}{l}\text { UA: S } \\
\text { MA: NI }\end{array}$ & $\begin{array}{c}\text { UA: OR: } 3.85 \\
\text { (CI: } 1.17-12.73) \\
\text { MA: NI }\end{array}$ \\
\hline $\begin{array}{l}\text { Shuto et al. } \\
\text { (2010) }\end{array}$ & Japan & 349 fallers & $71.5 \pm 14.8$ & 2,5 years & Case-crossover & $\begin{array}{l}\text { r Not mentioned or } \\
\text { evaluated }\end{array}$ & Antihypertensives & $\begin{array}{c}\text { amlodipine, atenolol, } \\
\text { benidipine, betaxolol, } \\
\text { bisoprolol, candesartan, } \\
\text { captopril, carvedilol, clonidina } \\
\text {, diltiazem, doxazosin, } \\
\text { efonidipine, enalapril, imidapril } \\
\text { losartan, etoprolol, nicardipine, } \\
\text { nifedipine, nilvadipine, } \\
\text { nisoldipine, perindopril, } \\
\text { prazosin, propranolol, } \\
\text { temocapril, valsartan, } \\
\text { verapamil }\end{array}$ & $\begin{array}{l}\text { UA: NI } \\
\text { MA: } \mathrm{S} \\
\text { MA for candersartan: }\end{array}$ & $\begin{array}{c}\text { UA: NI } \\
\text { MA: OR: } 8.42 \\
\text { (CI: } 3.12-22.72 \text { ) } \\
\text { MA for candersartan: } \\
\text { OR: } 13.92 \\
\text { (CI: } 1.71-113.69 \text { ) }\end{array}$ \\
\hline & & & & & & & $\begin{array}{l}\text { Anti-Parkinson } \\
\text { drugs }^{2}\end{array}$ & $\begin{array}{l}\text { amantadine, biperiden, } \\
\text { cabergoline, droxidope, } \\
\text { levodopa, pergolid), } \\
\text { pramipexole,selegiline, } \\
\text { tiapride, trihexifenidil }\end{array}$ & $\begin{array}{c}\text { UA: NI } \\
\text { MA: } \mathrm{S} \\
\text { MA for biperiden: } \mathrm{S}\end{array}$ & $\begin{array}{c}\text { UA: NI } \\
\text { MA: OR: } 4,18 \\
\text { (CI: } 1.75-10.02 \text { ) }\end{array}$ \\
\hline & & & & & & & & & & $\begin{array}{l}\text { MA for biperiden: OR } \\
4.34 \text { (IC: } 1.57 \text { - 11.99) }\end{array}$ \\
\hline & & & & & & & Anxiolytics & $\begin{array}{c}\text { alprazolam, bromazepam, } \\
\text { clotiazepam,cloxazolam, } \\
\text { diazepam, etil loflazepate, } \\
\text { etizolam, lorazepam, } \\
\text { tandospirona }\end{array}$ & $\begin{array}{c}\text { UA: NI } \\
\text { MA: S } \\
\text { MA for etizolam: S }\end{array}$ & $\begin{array}{c}\text { UA: NI } \\
\text { MA: OR: } 3.25 \text { (IC: } \\
1.62-6.50 \text { ) } \\
\text { MA for etizolam: OR: } \\
\text { 6.83 (IC: } 1.92-24.26 \text { ) }\end{array}$ \\
\hline & & & & & & & $\begin{array}{l}\text { Hypnotics and } \\
\text { sedatives }\end{array}$ & $\begin{array}{l}\text { brotizolam, flunitrazepam, } \\
\text { lormetazepam),midazolam, } \\
\text { nitrazepam, quazepam, } \\
\text { rilmazafone, triazolam, } \\
\text { zolpidem, zopiclone }\end{array}$ & $\begin{array}{c}\text { UA: NI } \\
\text { MA: S } \\
\text { MA for zopiclone: S }\end{array}$ & $\begin{array}{c}\text { UA: NI } \\
\text { MA:;OR: } 2.44 \\
\text { (CI: } 1.32-4.51)\end{array}$ \\
\hline & & & & & & & & & & $\begin{array}{l}\text { MA for zopiclone: } \\
\text { OR: } 4.20 \\
\text { (CI: } 1.55-11.40)\end{array}$ \\
\hline $\begin{array}{l}\text { Mamun, Lim } \\
\text { (2009) }\end{array}$ & Singapore & $\begin{array}{c}298 \text { fallers/ } 298 \\
\text { control; Asian }\end{array}$ & 75.8 & 12 months & Case control & $\begin{array}{l}\text { Normal mental } \\
\text { state on admission, }\end{array}$ & Anxiolytics & $\begin{array}{l}\text { benzodiazepines and } \\
\text { anxiolytics }\end{array}$ & & \\
\hline & & elderly & & & & length of stay, & & & MA: S & MA: NI \\
\hline & & & & & & $\begin{array}{l}\text { Morse scale, } \\
\text { history of } \\
\text { falls, walking } \\
\text { jundenendently }\end{array}$ & Cough supressant & Cough preparations NE & $\begin{array}{l}\text { UA: S } \\
\text { MA: S }\end{array}$ & $\begin{array}{l}\text { UA: NI } \\
\text { MA: NI }\end{array}$ \\
\hline & & & & & & $\begin{array}{l}\text { and number of } \\
\text { medications }\end{array}$ & $\begin{array}{l}\text { Antithrombotic } \\
\text { agents }\end{array}$ & Anti-platelet agents NE & UA: S & UA: NI \\
\hline & & & & & & & & & MA: S & MA: NI \\
\hline & & & & & & & $\begin{array}{l}\text { Vasodilators used } \\
\text { for treating heart- } \\
\text { related diseases }\end{array}$ & Nitrates & $\begin{array}{l}\text { UA: S } \\
\text { MA: NI }\end{array}$ & $\begin{array}{l}\text { UA: NI } \\
\text { MA: NI }\end{array}$ \\
\hline & & & & & & & $\begin{array}{l}\text { Calcium channel } \\
\text { blockers }^{2}\end{array}$ & Calcium channel blockers & UA: S & UA: NI \\
\hline & & & & & & & & & MA: NI & MA: NI \\
\hline & & & & & & & Opioids & Weak opioids & UA: S & UA: NI \\
\hline & & & & & & & & & MA: NI & MA: NI \\
\hline & & & & & & & Diuretics $^{2}$ & Diuretics NE & UA: S & UA: NI \\
\hline & & & & & & & & & MA: NI & MA: NI \\
\hline
\end{tabular}


TABLE III - Eligible studies data (cont.)

\begin{tabular}{|c|c|c|c|c|c|c|c|c|c|c|}
\hline $\begin{array}{l}\text { Author, } \\
\text { publication } \\
\text { year }\end{array}$ & Country & $\begin{array}{c}\mathbf{n} \text { fallers/ } \mathbf{n} \\
\text { control group } \\
\text { (or comparative } \\
\text { group); Special } \\
\text { sample condition } \\
\end{array}$ & $\begin{array}{l}\text { Median age } \\
\text { (fallers) }\end{array}$ & $\begin{array}{c}\text { Follow-up } \\
\text { period }\end{array}$ & Type of study & $\begin{array}{c}\text { Other factors } \\
\text { related to fall } \\
\text { (statistically } \\
\text { significant) }\end{array}$ & $\begin{array}{c}\text { ATC - } \\
\text { Pharmacological } \\
\text { subgroup }\end{array}$ & Medication & $\begin{array}{c}\text { Pvalue } \\
\text { Univariate } \\
\text { analysis (UA) } \\
\text { and Multivariate } \\
\text { analysis (MA) } \\
\end{array}$ & $\begin{array}{c}\text { Odds ratio } \\
\text { Univariate } \\
\text { analysis (UA) and } \\
\text { Multivariate analysis } \\
\text { (MA) } \\
\end{array}$ \\
\hline \multirow[t]{8}{*}{$\begin{array}{l}\text { Mamun, Lim } \\
\text { (2009) }\end{array}$} & \multirow[t]{8}{*}{ Singapore } & \multirow{8}{*}{$\begin{array}{c}298 \text { fallers } / 298 \\
\text { control; Asian } \\
\text { elderly }\end{array}$} & \multirow[t]{8}{*}{75.8} & \multirow[t]{8}{*}{12 months } & \multirow[t]{8}{*}{ Case control } & \multirow{8}{*}{$\begin{array}{c}\text { Normal mental } \\
\text { state on admission } \\
\text { length of stay, } \\
\text { Morse scale, } \\
\text { history of } \\
\text { falls, walking } \\
\text { independently } \\
\text { and number of } \\
\text { medications }\end{array}$} & $\begin{array}{l}\text { Lipid- modifying } \\
\quad \text { agents. plain }\end{array}$ & Lipid -regulating drugs $\mathrm{NE}$ & UA: $\mathrm{S}$ & UA: NI \\
\hline & & & & & & & & & MA: NI & MA: NI \\
\hline & & & & & & & $\begin{array}{c}\text { Beta-blocking } \\
\text { Agents }\end{array}$ & Beta-blockers & UA: $\mathrm{S}$ & UA: NI \\
\hline & & & & & & & & & MA: NI & MA: NI \\
\hline & & & & & & & $\begin{array}{l}\text { Alpha and beta } \\
\text { adrenoreceptor }\end{array}$ & Alpha-agonist & UA: $S$ & UA: NI \\
\hline & & & & & & & agonist & & MA: NI & MA: NI \\
\hline & & & & & & & $\begin{array}{l}\text { Corticosteroids for } \\
\text { systemic use }\end{array}$ & Steroides & UA: $S$ & UA: NI \\
\hline & & & & & & & & & MA: NI & MA: NI \\
\hline \multirow[t]{10}{*}{$\begin{array}{l}\text { Tanaka et al. } \\
(2008)\end{array}$} & \multirow[t]{10}{*}{ Japan } & \multirow[t]{10}{*}{$\begin{array}{c}65 \text { fallers/ } 4084 \\
\text { control; NA }\end{array}$} & \multirow[t]{10}{*}{$68.1 \pm 13.1$} & \multirow[t]{10}{*}{7 months } & \multirow[t]{10}{*}{ Case control } & \multirow{10}{*}{$\begin{array}{c}\text { Age }>70 \\
\text { years. Other } \\
\text { non-specified } \\
\text { conditions. }\end{array}$} & Anxiolytics & Anxiolytics NE & $\begin{array}{l}\text { UA: S } \\
\text { MA: S }\end{array}$ & $\begin{array}{l}\text { UA: OR: } 3.35 \\
\text { (CI: } 1.83-5.82)\end{array}$ \\
\hline & & & & & & & & & & $\begin{array}{l}\text { MA: OR: } 2.36 \\
\text { (CI: } 1.24-4.28)\end{array}$ \\
\hline & & & & & & & $\begin{array}{l}\text { Anti-Parkinson } \\
\text { drugs }\end{array}$ & Anti-Parkinson drugs NE & UA: NI & $\begin{array}{c}\text { UA: OR: } 5.7 \\
\text { (CI: } 1.71-14.80)\end{array}$ \\
\hline & & & & & & & & & MA: S & $\begin{array}{l}\text { MA: OR: } 5.04 \\
\text { (CI: } 1.44-13.43)\end{array}$ \\
\hline & & & & & & & $\begin{array}{l}\text { Hypnotics and } \\
\text { sedatives }\end{array}$ & Hypnotics and sedatives NE & UA: S & $\begin{array}{l}\text { UA: OR:2.12 } \\
\text { (CI: } 1.25-3.52)\end{array}$ \\
\hline & & & & & & & & & MA: NS & MA: NI \\
\hline & & & & & & & Opioids & Opioids NE & & $\begin{array}{l}\text { UA: OR: } 3.08 \text {. } \\
\text { (CI: } 1.06-7.11 \text { ) }\end{array}$ \\
\hline & & & & & & & & & MA: NS & MA: NI \\
\hline & & & & & & & Diuretics $^{2}$ & Diuretics NE & & $\begin{array}{l}\text { UA: OR: } 2.39 \\
\text { (CI: } 1.42-3.95)\end{array}$ \\
\hline & & & & & & & & & MA: NI & MA: NI \\
\hline \multirow{5}{*}{$\begin{array}{l}\text { Angalakuditi, } \\
\text { Gomes, Coley } \\
\text { (2007) }\end{array}$} & \multirow[t]{5}{*}{ US } & \multirow{5}{*}{$\begin{array}{l}635 \text { fallers/ } 1270 \\
\text { control; With } \\
\text { chronic kidney } \\
\text { disease }\end{array}$} & \multirow[t]{5}{*}{$68 \pm 15$} & \multirow[t]{5}{*}{5.5 years } & \multirow[t]{5}{*}{ Case control } & \multirow[t]{5}{*}{$\begin{array}{c}\text { Dementia, diabetes } \\
\text { and pneumonia. }\end{array}$} & s Antiepileptics & Anticonvulsant NE & UA: S & UA: NI \\
\hline & & & & & & & & & MA: S & $\begin{array}{l}\text { MA: OR: } 1.52 \\
(\mathrm{CI}: 1.13-2.03) \\
\end{array}$ \\
\hline & & & & & & & Antidepressants & Antidepressants NE & $\begin{array}{l}\text { UA: S } \\
\text { MA: S }\end{array}$ & $\begin{array}{c}\text { UA: NI } \\
\text { MA: OR: } 1.65 \\
\text { (CI: } 1.32-2.08) \\
\end{array}$ \\
\hline & & & & & & & Anxiolytics & Benzodiazepines NE & UA:S & UA: NI \\
\hline & & & & & & & & & MA: S & $\begin{array}{l}\text { MA: OR: } 0.69 \\
\text { (CI: } 0.55-0.86)\end{array}$ \\
\hline \multirow[t]{6}{*}{$\begin{array}{l}\text { Vassalo et al. } \\
\text { (2006) }\end{array}$} & \multirow[t]{6}{*}{ UK } & \multirow[t]{6}{*}{$\begin{array}{c}825 \text { falls; } \\
\text { Confused patients }\end{array}$} & \multirow[t]{6}{*}{81.9} & \multirow[t]{6}{*}{17 months } & \multirow[t]{6}{*}{ Case control } & $\begin{array}{l}\text { Confused patients. } \\
\text { Others not } \\
\text { evaluated. }\end{array}$ & Anxiolytics & Benzodiazepines NE & $\begin{array}{c}\text { UA for falls on } \\
\text { tranquilizers } \\
\text { (benzodiazepines } \\
\text { and antipsychotics): } \\
\text { S }\end{array}$ & $\begin{array}{c}\text { UA for falls on } \\
\text { tranquilizers } \\
\text { (benzodiazepines } \\
\text { and antipsychotics): } \\
\text { OR: } 0.63 \\
\text { (CI: } 047-0.82 \text { ) }\end{array}$ \\
\hline & & & & & & & & & $\begin{array}{l}\text { UA for recurrent } \\
\text { falls in confused: } \mathrm{S}\end{array}$ & $\begin{array}{l}\text { UA for recurrent falls } \\
\text { in confused: NI }\end{array}$ \\
\hline & & & & & & & & & MA: NI & MA: NI \\
\hline & & & & & & & Antipsychotics & Antipsychotics NE & $\begin{array}{c}\text { UA for falls on } \\
\text { tranquilizers } \\
\text { (benzodiazepines } \\
\text { and antipsychotics): } \\
\text { S }\end{array}$ & $\begin{array}{c}\text { UA for falls on } \\
\text { tranquilizers } \\
\text { (benzodiazepines } \\
\text { and antipsychotics): } \\
\text { OR: } 0.63 \\
\text { (CI: } 047-0.82 \text { ) }\end{array}$ \\
\hline & & & & & & & & & $\begin{array}{l}\text { UA for recurrent } \\
\text { falls in confused: } \mathrm{S}\end{array}$ & $\begin{array}{l}\text { UA for recurrent falls } \\
\text { in confused: NI }\end{array}$ \\
\hline & & & & & & & & & MA: NI & MA: NI \\
\hline Walker (2005) & US & $\begin{array}{c}62 \text { fallers/ } 62 \\
\text { control; NA }\end{array}$ & $74.54 \pm 6.03$ & 12 months & Case control & $\begin{array}{c}\text { Mean } \pm \text { S.D. } \\
\text { no. medications }\end{array}$ & $\begin{array}{c}\text { Other Analgesics e } \\
\text { Antipyretics }\end{array}$ & $\begin{array}{l}\text { Aspirin } 85 \text { e } 325(77 \%) . \\
\text { aspirin+celecoxibe. }\end{array}$ & UA: $\mathrm{S}$ & $\begin{array}{l}\text { UA: OR: } 10.02 \\
(2.6-38.58)\end{array}$ \\
\hline & & & & & & $\begin{array}{l}\text { received within } 24 \\
\text { hr preceding fall } \\
\text { and Dementia. }\end{array}$ & & $\begin{array}{c}\text { aspirin }+ \text { ibuprofen, celecoxib, } \\
\text { ibuprofen, ketorolac, } \\
\text { indometacin }\end{array}$ & MA: NI & MA: NI \\
\hline & & & & & & & Opioids & Opioids NE & UA: S & $\begin{array}{c}\text { UA:OR: } 0.33 \text { (IC: } \\
0.11-0.96)\end{array}$ \\
\hline & & & & & & & & & MA: NI & MA: NI \\
\hline
\end{tabular}


Medication-related inpatient falls: a critical review

TABLE III - Eligible studies data (cont.)

\begin{tabular}{|c|c|c|c|c|c|c|c|c|c|c|}
\hline $\begin{array}{l}\text { Author, } \\
\text { publication } \\
\text { year }\end{array}$ & Country & $\begin{array}{c}\mathbf{n} \text { fallers/ } \mathbf{n} \\
\text { control group } \\
\text { (or comparative } \\
\text { group); Special } \\
\text { sample condition }\end{array}$ & $\begin{array}{l}\text { Median age } \\
\text { (fallers) }\end{array}$ & $\begin{array}{c}\text { Follow-up } \\
\text { period }\end{array}$ & Type of study & $\begin{array}{c}\text { Other factors } \\
\text { related to fall } \\
\text { (statistically } \\
\text { significant) }\end{array}$ & $\begin{array}{c}\text { ATC - } \\
\text { Pharmacological } \\
\text { subgroup }\end{array}$ & Medication & $\begin{array}{c}\text { Pvalue } \\
\text { Univariate } \\
\text { analysis (UA) } \\
\text { and Multivariate } \\
\text { analysis (MA) } \\
\end{array}$ & $\begin{array}{c}\text { Odds ratio } \\
\text { Univariate } \\
\text { analysis (UA) and } \\
\text { Multivariate analysis } \\
\text { (MA) } \\
\end{array}$ \\
\hline \multirow[t]{12}{*}{ Walker (2005) } & \multirow[t]{12}{*}{ US } & \multirow[t]{12}{*}{$\begin{array}{c}62 \text { fallers/ } 62 \\
\text { control; NA }\end{array}$} & \multirow[t]{12}{*}{$74.54 \pm 6.03$} & \multirow[t]{12}{*}{12 months } & \multirow[t]{12}{*}{ Case control } & \multirow[t]{12}{*}{$\begin{array}{l}\text { Mean } \pm \text { S.D. } \\
\text { no. medications } \\
\text { received within } 24 \\
\text { hr preceding fall } \\
\text { and Dementia. }\end{array}$} & Urological drugs & \multirow[t]{2}{*}{ Oxybutynin and tolterodine } & $\begin{array}{l}\text { UA for other } \\
\text { medication that } \\
\text { can produce } \\
\text { sedation or postural } \\
\text { hypotension: } \mathrm{S}\end{array}$ & $\begin{array}{c}\text { UA for other } \\
\text { medication that can } \\
\text { produce sedation or } \\
\text { postural hypotension: } \\
\text { OR: } 13.85 \text { (IC:3.6- } \\
52.83 \text { ) }\end{array}$ \\
\hline & & & & & & & & & MA: NI & MA: NI \\
\hline & & & & & & & $\begin{array}{c}\text { Blood glucose } \\
\text { lowering drugs, } \\
\text { excluding insulin }\end{array}$ & $\begin{array}{l}\text { glimepiride, glipizide, insulin, } \\
\text { rosiglitazone }\end{array}$ & $\begin{array}{l}\text { UA for other } \\
\text { medication that } \\
\text { can produce } \\
\text { sedation or postural } \\
\text { hypotension: } \mathrm{S}\end{array}$ & $\begin{array}{c}\text { UA for other } \\
\text { medication that can } \\
\text { produce sedation or } \\
\text { postural hypotension: } \\
\text { OR: } 13.85 \text { (IC:3.6- } \\
52.83 \text { ) }\end{array}$ \\
\hline & & & & & & & \multicolumn{3}{|r|}{ MA: NI } & \\
\hline & & & & & & & \multirow[t]{2}{*}{ Antiepileptics } & \multirow[t]{2}{*}{$\begin{array}{c}\text { carbamazepine, gabapentin, } \\
\text { lamotrigine, oxcarbazine, } \\
\text { phenytoin, topiramate, } \\
\text { valproate. }\end{array}$} & $\begin{array}{l}\text { UA for other } \\
\text { medication that } \\
\text { can produce } \\
\text { sedation or postural } \\
\text { hypotension: } \mathrm{S}\end{array}$ & $\begin{array}{c}\text { UA for other } \\
\text { medication that can } \\
\text { produce sedation or } \\
\text { postural hypotension: } \\
\text { OR: } 13.85 \text { (IC:3.6- } \\
52.83 \text { ) }\end{array}$ \\
\hline & & & & & & & & & MA: NI & MA: NI \\
\hline & & & & & & & $\begin{array}{l}\text { Antihistamines for } \\
\text { systemic use }\end{array}$ & cyclizine, fexofenadine & $\begin{array}{l}\text { UA for other } \\
\text { medication that } \\
\text { can produce } \\
\text { sedation or postural } \\
\text { hypotension: } \mathrm{S}\end{array}$ & $\begin{array}{c}\text { UA for other } \\
\text { medication that can } \\
\text { produce sedation or } \\
\text { postural hypotension: } \\
\text { OR: } 13.85 \text { (IC:3.6- } \\
52.83 \text { ) }\end{array}$ \\
\hline & & & & & & & \multicolumn{3}{|r|}{ MA: NI } & MA: NI \\
\hline & & & & & & & \multirow[t]{2}{*}{$\begin{array}{c}\text { Drugs for } \\
\text { alimentary tract } \\
\text { and metabolism }\end{array}$} & \multirow[t]{2}{*}{$\begin{array}{l}\text { dolasetron, metoclopramide, } \\
\text { omeprazole, ondansetron, } \\
\text { ranitidine }\end{array}$} & $\begin{array}{l}\text { UA for other } \\
\text { medication that } \\
\text { can produce } \\
\text { sedation or postural } \\
\text { hypotension: } \mathrm{S}\end{array}$ & $\begin{array}{c}\text { UA for other } \\
\text { medication that can } \\
\text { produce sedation or } \\
\text { postural hypotension: } \\
\text { OR: } 13.85 \text { (IC:3.6- } \\
52.83 \text { ) }\end{array}$ \\
\hline & & & & & & & & & MA: NI & MA: NI \\
\hline & & & & & & & \multirow[t]{2}{*}{$\begin{array}{c}\text { Drugs for } \\
\text { Cardiovascular } \\
\text { system }^{2}\end{array}$} & \multirow[t]{2}{*}{ Amlodipine, diltiazem, nitrates } & $\begin{array}{l}\text { UA for other } \\
\text { medication that } \\
\text { can produce } \\
\text { sedation or postural } \\
\text { hypotension: } \mathrm{S}\end{array}$ & $\begin{array}{c}\text { UA for other } \\
\text { medication that can } \\
\text { produce sedation or } \\
\text { postural hypotension: } \\
\text { OR: } 13.85 \text { (IC:3.6- } \\
52.83 \text { ) }\end{array}$ \\
\hline & & & & & & & & & MA: NI & MA: NI \\
\hline \multirow[t]{6}{*}{$\begin{array}{l}\text { Krauss et al. } \\
(2005)\end{array}$} & \multirow[t]{6}{*}{ US } & \multirow[t]{6}{*}{$\begin{array}{c}98 \text { fallers/ } 318 \\
\text { controls; NA }\end{array}$} & \multirow[t]{6}{*}{$\begin{array}{c}\text { ND }(50-69 \\
\text { years }-37.8 \%) \\
\text { and }>=70 \\
(38.8 \%)\end{array}$} & \multirow[t]{6}{*}{6 weeks } & \multirow[t]{6}{*}{ Case control } & \multirow{6}{*}{$\begin{array}{l}\text { Age }>50 \text { years, } \\
\text { gait/balance deficit } \\
\text { or lower extremity, } \\
\text { incontinence, } \\
\text { diabetes, fallen in } \\
\text { the past } 6 \text { months, } \\
\text { depression, } \\
\text { impaired memory, } \\
\text { confused, and } \\
\text { care-related } \\
\text { factors (assistance, } \\
\text { patient-to-nurse } \\
\text { ration) }\end{array}$} & $\begin{array}{l}\text { Hypnotics and } \\
\text { sedatives }\end{array}$ & Hypnotics and sedatives NE & $\begin{array}{l}\text { UA: NI } \\
\text { MA: NI }\end{array}$ & $\begin{array}{l}\text { UA: OR: } 2.1 \\
\text { (CI: } 1.2-3.7) \\
\text { MA: OR: } 4.3 \\
\text { (CI: } 1.6-11.5)\end{array}$ \\
\hline & & & & & & & $\begin{array}{l}\text { Antiarrhythmics } \\
\text { class I and III }\end{array}$ & $\begin{array}{l}\text { Antiarrhythmics class I and } \\
\text { III NE }\end{array}$ & $\begin{array}{l}\text { UA: NI } \\
\text { MA: NI }\end{array}$ & $\begin{array}{c}\text { UA: OR: } 2.1 \\
\text { (CI }: 1.2-3.7) \\
\text { MA: NI }\end{array}$ \\
\hline & & & & & & & Diabetic drugs $^{2}$ & Diabetic drugs NE & & $\begin{array}{l}\text { UA: OR: } 2.1 \\
\text { (CI: } 1.2-3.5)\end{array}$ \\
\hline & & & & & & & & & MAA. NI & $\begin{array}{l}\text { MA: OR: } 3.2 \\
\text { (CI: } 1.3-7.9)\end{array}$ \\
\hline & & & & & & & $\begin{array}{l}\text { Other Analgesics e } \\
\text { Antipyretics }\end{array}$ & Non-narcotic analgesics - NE & UA: NI & $\begin{array}{l}\text { UA: OR:2.0 } \\
\text { (CI:0.9-3.3) }\end{array}$ \\
\hline & & & & & & & & & MA: NI & MA: NI \\
\hline $\begin{array}{l}\text { Vassalo et al. } \\
\text { (2004) }\end{array}$ & UK & $\begin{array}{l}136 \text { fallers/ } 463 \\
\text { control ; With } \\
\text { unsafe-safe gait }\end{array}$ & 81.9 & 12 months & $\begin{array}{l}\text { Prospective } \\
\text { Cohort }\end{array}$ & $\begin{array}{l}\text { Unsafe gait, } \\
\text { previous fall and }\end{array}$ & Anxiolytics & Tranquilizers NE & & $\begin{array}{l}\text { UA: OR: } 1.73 \\
\text { (CI:1.08-2.75) }\end{array}$ \\
\hline & & unsafe-safe gait & & & & confusion & & & MA: NI & MA: NI \\
\hline Frels (2002) & UK & $\begin{array}{l}181 \text { fallers/ } 181 \\
\text { controls; Elderly }\end{array}$ & 73.3 & 4 months & Case control & $\begin{array}{l}\text { Stroke, previous } \\
\text { fall, disoriented }\end{array}$ & Anxiolytics & Benzodiazepines NE & UA: S & UA: NI \\
\hline & & & & & & $\begin{array}{l}\text { in time/place } \\
\text { or person and }\end{array}$ & & & MA: S & $\begin{array}{l}\text { MA: OR: } 2.3 \\
(\mathrm{CI}: 1.4-3.7)\end{array}$ \\
\hline & & & & & & $\begin{array}{l}\text { needs maxımum } \\
\text { assistance. }\end{array}$ & Diuretics $^{2}$ & Diuretics NE & UA: S & UA: NI \\
\hline & & & & & & & & & MA: NS & MA: NI \\
\hline
\end{tabular}


TABLE III - Eligible studies data (cont.)

\begin{tabular}{|c|c|c|c|c|c|c|c|c|c|c|}
\hline $\begin{array}{l}\text { Author, } \\
\text { publication } \\
\text { year }\end{array}$ & Country & $\begin{array}{l}\mathbf{n} \text { fallers/ } \mathbf{n} \\
\text { control group } \\
\text { (or comparative } \\
\text { group); Special } \\
\text { sample condition }\end{array}$ & $\begin{array}{l}\text { Median age } \\
\text { (fallers) }\end{array}$ & $\begin{array}{l}\text { Follow-up } \\
\text { period }\end{array}$ & Type of study & $\begin{array}{c}\text { Other factors } \\
\text { related to fall } \\
\text { (statistically } \\
\text { significant) }\end{array}$ & $\begin{array}{c}\text { ATC - } \\
\text { Pharmacological } \\
\text { subgroup }\end{array}$ & Medication & $\begin{array}{c}\text { Pvalue } \\
\text { Univariate } \\
\text { analysis (UA) } \\
\text { and Multivariate } \\
\text { analysis (MA) }\end{array}$ & $\begin{array}{c}\text { Odds ratio } \\
\text { Univariate } \\
\text { analysis (UA) and } \\
\text { Multivariate analysis } \\
\text { (MA) }\end{array}$ \\
\hline \multirow[t]{10}{*}{$\begin{array}{l}\text { Mendelson } \\
\text { (1996) }\end{array}$} & \multirow[t]{10}{*}{ US } & \multirow[t]{10}{*}{$\begin{array}{l}253 \text { falls/ } 84 \\
\text { control; NA }\end{array}$} & \multirow[t]{10}{*}{$57.4 \pm 1.3$} & \multirow[t]{10}{*}{12 months } & \multirow[t]{10}{*}{ Case control } & \multirow[t]{10}{*}{$\begin{array}{l}\text { Not mentioned or } \\
\text { evaluated. }\end{array}$} & Antidepressants & $\begin{array}{c}\text { Amitriptyline.clomipramine, } \\
\text { doxepin, fluoxetine, } \\
\text { imipramine, nortriptyline and } \\
\text { sertraline }\end{array}$ & $\begin{array}{c}\text { UA for } \\
\text { antidepressants: } \mathrm{S} \\
\text { UA for Nortriptyline: } \\
\text { S }\end{array}$ & $\begin{array}{l}\text { UA: NI } \\
\text { UA for Nortriptyline: } \\
\text { NI }\end{array}$ \\
\hline & & & & & & & & & UA for sertraline: $\mathrm{S}$ & UA for sertraline: NI \\
\hline & & & & & & & & & MA: NI & MA: NI \\
\hline & & & & & & & $\begin{array}{l}\text { Hypnotics and } \\
\text { sedatives }\end{array}$ & $\begin{array}{c}\text { Chloral hydrate, temazepam } \\
\text { andtriazolam }\end{array}$ & UA for hypnotics: S & UA for hypnotics: NI \\
\hline & & & & & & & & & UA for temazepam:S & $\begin{array}{c}\text { UA for } \\
\text { temazepam:NI }\end{array}$ \\
\hline & & & & & & & & & MA: NI & MA: NI \\
\hline & & & & & & & Anxiolytics & $\begin{array}{c}\text { Alprozolam,chlordiazepoxide, } \\
\text { Chlorazepate, diazepam e } \\
\text { lorazepam }\end{array}$ & $\begin{array}{c}\text { UA for } \\
\text { benzodiazepines: } \mathrm{S}\end{array}$ & $\begin{array}{c}\text { UA for } \\
\text { benzodiazepines: NI }\end{array}$ \\
\hline & & & & & & & & & $\begin{array}{l}\text { UA for alprozolam. } \\
\text { diazepam and } \\
\text { lorazepam: S }\end{array}$ & $\begin{array}{l}\text { UA for alprozolam. } \\
\text { diazepam and } \\
\text { lorazepam: NI }\end{array}$ \\
\hline & & & & & & & & & MA: NI & MA: NI \\
\hline & & & & & & & Antipsychotics & $\begin{array}{l}\text { Clorpromazine, droperidol. } \\
\text { Haloperidol,prochlorperazinam, } \\
\text { Tioridazinam,tiotixene and } \\
\text { trifluoerazine }\end{array}$ & $\begin{array}{c}\text { UA for general: } \mathrm{S} \\
\text { UA for } \\
\text { haloperidol and } \\
\text { prochlorperazine: } \mathrm{S}\end{array}$ & $\begin{array}{l}\text { UA for general: NI } \\
\text { UA for haloperidol and } \\
\text { prochlorperazine: NI }\end{array}$ \\
\hline \multirow[t]{4}{*}{$\begin{array}{l}\text { Aisen, Deluca, } \\
\text { Lawlor (1992) }\end{array}$} & \multirow[t]{4}{*}{ UK } & \multirow{4}{*}{$\begin{array}{c}33 \text { falls ( } 17 \\
\text { fallers)/ } 30 \text { control; } \\
\text { Geropsychiatry } \\
\text { inpatients }\end{array}$} & \multirow[t]{4}{*}{$75.3 \pm 7$} & \multirow[t]{4}{*}{12 months } & \multirow[t]{4}{*}{ Case control } & \multirow{4}{*}{$\begin{array}{l}\text { Length of stay, } \\
\text { dementia, history } \\
\text { of falls and } \\
\text { assistance required }\end{array}$} & Anxiolytics & Benzodiazepines NE & UA: S & UA: NI \\
\hline & & & & & & & & & MA: NI & MA: NI \\
\hline & & & & & & & Antipsychotics & Neuroleptics NE & UA: S & UA: NI \\
\hline & & & & & & & & & MA: NI & MA: NI \\
\hline
\end{tabular}

Legend: ATC: Anatomical Therapeutic Chemical Code; CI: confidence interval 95\%; CNS meds: Central nervous system medication; MA: multivariate analysis; "n": number of participants; NA: not applicable; NE: nonspecified; NI: not informed; OR: odds ratio; UA: univariate analysis. "1":Psychotropics could not be classified according to pharmacological subgroup; "2": These medications were classified according to ATC Therapeutic Subgroup $\left(2^{\text {nd }}\right.$ level $)$.

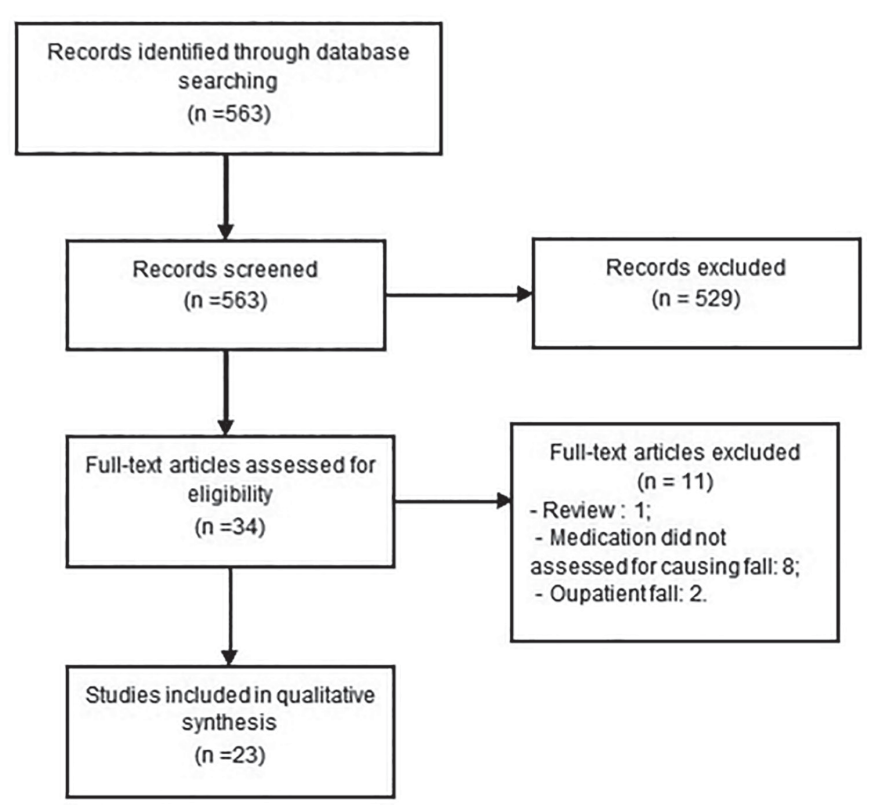

FIGURE 1 - Flowchart of studies selection according to PRISMA, 2009.

of the study designs covered case controls, $18 \%$ dealt with retrospective cohorts $(4 / 23), 13 \%$ were focused on prospective cohorts $(3 / 23)$, one study was cross- sectional and the remaining one was case-crossover. The retrospective studies represented $87 \%(20 / 23)$ of the total number of eligible full studies. No randomized controlled trial (RCT) was found. Studies performed in a single institution represented $91 \%(21 / 23)$ of the total number of studies under investigation.

The follow-up period for patient observation in almost half of the studies lasted 12 months; in 30\% $(7 / 23)$ of the studies the follow-up period was less than 12 months; and four studies reported follow-up periods longer than one year (1.5 to 5.5 years).

The total number of inpatients in the studies amounted to 27,449 ; this consisted of 4,474 fallers and 22,975 classified as control or comparative group. The median age of the fallers was 69 years. Another fact that deserves mentioning is that in four studies (17\%) the population was restricted to only elderly patients. Six other studies included special population: cirrhotic patient, patients with unsafe gait, patients who are exposed to anticholinergic and sedatives drugs, psychiatric patients with hyponatremia, geropsychiatric patients and those with chronic kidney disease.

Fifty seven percent of the studies did not specify the medication, class or substances included in the 
group (only mentioned, for instance, sedative hypnotics, without indicating any specific drug) (Costa-Dias et al., 2014; Pierce Jr. et al., 2013; Obayashi et al., 2013; Lamis et al., 2012; Chang et al., 2011; Bun, Serby, Friedmann, 2011; Mamun, Lim, 2009; Tanaka et al., 2008; Angalakuditi, Gomes, Coley, 2007; Vassalo et al., 2006; Krauss et al., 2005; Vassalo et al., 2004; Aisen, Deluca, Lawlor, 1992). Some articles focused their studies on all central nervous system medications (Lamis et al., 2012) (without establishing any distinction between the pharmacological groups). Some studies grouped more than one pharmacological group (Costa-Dias et al., 2014; Vassalo et al., 2006). Walker (2005) evaluated a broad class of medications and identified it as "other medications that can cause sedation and postural hypotension". They also included different pharmacological groups in their analysis (urological drugs; blood glucose lowering drugs excluding insulin; antiepileptics; antihistamines for systemic use; drugs for alimentary tract and metabolism; drugs for cardiovascular system). Dauphinot et al. (2014) evaluated defined daily dose (DDD) of anticholinergic and sedative agents. They found that DDD increase was related to fall, but made no assessment of the relation between the medication and the falling incidents.

Only six studies presented all confounders correctly (Figure 2). Other risk factors for inpatient fall were evaluated. These included age, oncologic patient, neurodegenerative disease, male gender, chronic/ palliative inpatient wards, previous falls, cognitive dysfunction, wheelchair use, patients in need of help to move, rehabilitation, patients in need of help with daily life activities, incontinence, diabetes, depression and impaired memory.

A decreasing list was made based on the studies and pharmacological subgroups (Table III). Some drugs were not classified in the pharmacological subgroup due to the fact that the study failed to specify the group to which these drugs belong. Hence, these drugs were exceptionally classified under the therapeutic subgroup ( $2^{\text {nd }}$ level by ATC classification) category: calcium channel blockers, diuretics, anti-parkinson drugs, antihypertensives, antidiabetic drugs, drugs for alimentary tract and metabolism, and drugs used for treating the cardiovascular system. Remarkably, there was only one study that used a classification which had no designation under the ATC classification system. The classification was that of unspecified psychotropic drugs.

In seventy-four percent of the studies (17/23), the inpatient falls were attributed to anxiolytics, hypnotics and sedatives accounted for $48 \%(11 / 23)$, antipsychotics represented $35 \%(8 / 23)$, opioids and antiepileptics

\begin{tabular}{|c|c|c|c|c|c|}
\hline Bias analysis / Author, year & \begin{tabular}{|l} 
Clear \\
population \\
definition \\
and \\
representa \\
tivity
\end{tabular} & $\begin{array}{l}\text { Selection of } \\
\text { control and } \\
\text { cases } \\
\text { (adequate } \\
\text { criteria and } \\
\text { sample) }\end{array}$ & \begin{tabular}{|l} 
Full \\
identificati \\
on of \\
potential \\
confounder \\
s
\end{tabular} & $\begin{array}{l}\text { Accurate } \\
\text { measure of } \\
\text { all relevant } \\
\text { exposure, } \\
\text { potential } \\
\text { confounder } \\
\text { s and } \\
\text { outcomes }\end{array}$ & $\begin{array}{l}\text { Adequate } \\
\text { statistical } \\
\text { analysis }\end{array}$ \\
\hline O'Neil et al., 2015 & 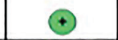 & $\odot$ & $\odot$ & $\odot$ & $\odot$ \\
\hline Tapper et ai. ,2015 & $\odot$ & $\odot$ & $\odot$ & $\odot$ & ? \\
\hline Hayakawa et al., 2014 & $\oplus$ & $\odot$ & $\odot$ & $\odot$ & $\odot$ \\
\hline Dauphinot et al., 2014 & $\odot$ & $\odot$ & $\oplus$ & $\odot$ & (?) \\
\hline Costa-Dias et al., 2014 & (2) & ? & $\odot$ & $\odot$ & $\odot$ \\
\hline Kolla et al., 2013 & 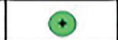 & $\odot$ & $\odot$ & $\odot$ & (?) \\
\hline Pierce Jr et $a l, 2013$ & 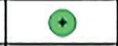 & $\odot$ & $\odot$ & $\odot$ & $\odot$ \\
\hline Obayashi et al., 2013 & 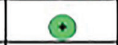 & $\odot$ & $\odot$ & $\odot$ & $\odot$ \\
\hline Lamis et al., 2012 & $\odot$ & + & $\odot$ & $\odot$ & $\odot$ \\
\hline Chang et at. 2011 & $\odot$ & + & $\odot$ & $\odot$ & $\odot$ \\
\hline Cashin et al, 2011 & $\odot$ & $\odot$ & $\odot$ & $\odot$ & $\odot$ \\
\hline Bun et al., 2011 & $\odot$ & $\odot$ & $\odot$ & ? & $\odot$ \\
\hline Shuto et al. 2010 & $\odot$ & (?) & $\odot$ & $\odot$ & (?) \\
\hline Mamun et ai., 2009 & $\odot$ & $\odot$ & $\odot$ & $\odot$ & (?) \\
\hline Tanaka et al., 2008 & $\odot$ & $\odot$ & $\odot$ & $\odot$ & $\odot$ \\
\hline Angalakuditi et al., 2007 & $\odot$ & $\odot$ & $\odot$ & $\odot$ & $\odot$ \\
\hline Vassalo et al., 2006 & $\odot$ & $\odot$ & $\odot$ & $\odot$ & ? \\
\hline Walker et al., 2005 & $\odot$ & $\odot$ & $\odot$ & $\odot$ & $\odot$ \\
\hline Krauss et al., 2004 & 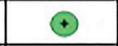 & 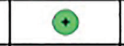 & $\odot$ & $\odot$ & ? \\
\hline Vassalo et al., 2004 & $\odot$ & 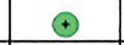 & $\odot$ & $\odot$ & $\odot$ \\
\hline Frels et al., 2002 & $\odot$ & $\odot$ & $\odot$ & $\oplus$ & (?) \\
\hline Mendelson et al., 1996 & $\odot$ & $\odot$ & $\odot$ & $\odot$ & $\odot$ \\
\hline Aisen et al., 1992 & $\odot$ & $\odot$ & $\odot$ & (3) & $\odot$ \\
\hline
\end{tabular}

Legend:

$$
\begin{aligned}
& + \text { Low risk of bias } \\
& \text { - High risk of bias } \\
& \text { ? Uncertain risk of bias }
\end{aligned}
$$

FIGURE 2 - Studies bias analysis (Tool adapted from STROBE (2007) and Young and Solomon (2009) proposition for critically appraise an article).

accounted for $30 \%(\mathrm{n}=7)$ while antidepressants represented $22 \%(5 / 23)$. Diuretics (4/23), anti-parkinson drugs (3/23), other analgesics and antipyretics $(3 / 23)$ were mentioned in less than $20 \%$ of the studies. The other subgroups accounted for less than $10 \%$ of inpatient falls in the studies.

The studies were also subjected to quality assessment using the bias analysis tool (Figure 2). The results obtained showed that only $26 \%(n=8)$ of the studies presented full identification of potential confounders, $52 \%$ had high risk of bias for selection of control and intervention cases and only $39 \%$ exhibited low risk of bias for statistical analysis. The pharmacological subgroups that were cited more 
frequently (in more than $20 \%$ of the eligible studies) are described below.

\section{Anxiolytics}

Seventeen studies attributed inpatient falls to anxiolytics use (Tapper, Risech-Neyman, Segupta, 2015; O'Neil et al., 2015; Mamun, Lim, 2009; Lamis et al., 2012; Frels, 2002; Dauphinot et al., 2014; Costa-Dias et al., 2014; Tanaka et al., 2008; Chang et al., 2011; Angalakuditi, Gomes, Coley, 2007; Shuto et al., 2010; Cashin, Yang, 2011; Vassalo et al., 2004; Mendelson,

TABLE IV - Distribution of pharmacological subgroups according to ATC classification for medications related to inpatient falls

Pharmacological Subgroup related to inpatient fall

Anxiolytics

Hypnotics and sedatives

Antipsychotics

Opioids

Antiepileptics

Antidepressants

Diuretics $^{2}$

Anti-Parkinson drugs ${ }^{2}$

Other Analgesics e Antipyretics

DiabetIC drugs ${ }^{2}$

Drugs for cardiovascular system ${ }^{2}$

Antipropulsives

Antihistamines for systemic use

Antiarrhythmic drugs - class I and III

Agents acting on the renin-angiotensin system

Urological drugs

Blood glucose lowering drugs, excluding insulin

Beta-blocking agentes

Drugs for alimentary tract and metabolism ${ }^{2}$

Alpha and beta adrenoreceptor agonist

Centrally-acting antiadrenergic agents

Anti-dementia drugs

Antihypertensives ${ }^{2}$

Antithrombotic agents

Blood glucose lowering drugs, excluding insulin

Calcium channel blockers ${ }^{2}$

Corticosteroids for systemic use

Cough supressant

Insulin and analogues

Lipid modifying agents, plain

Psychotropics $^{1}$

Vasodilators used for treating heart-related diseases
Studies " $n$ "

11

8

7

7

5

4

3

3

2

2

2

2

2

1

1

1

\section{1}

\section{1}

1

\section{1}

1

1

\section{1}

1

\section{1}

1

\section{1}

\section{1}

1

1

1

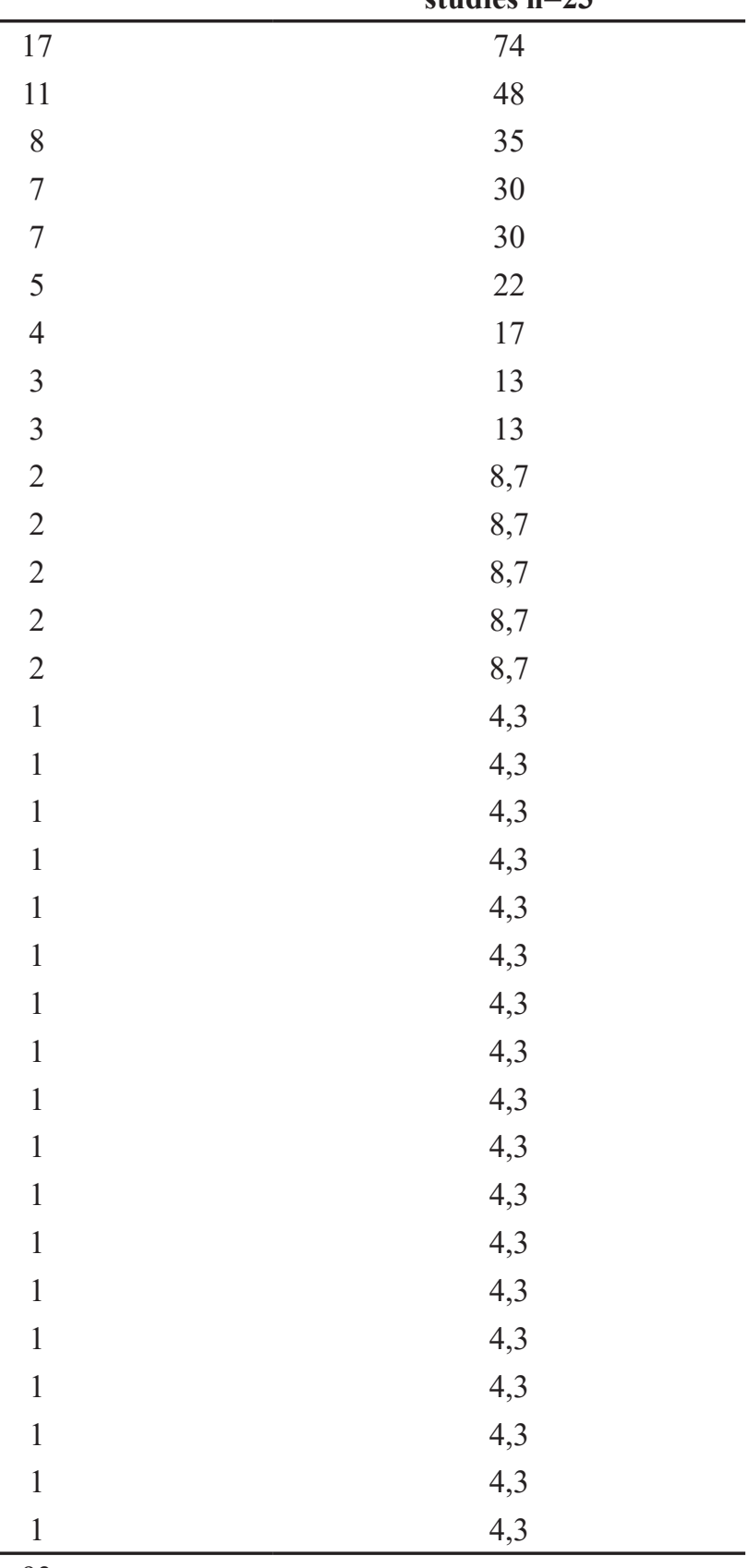

\section{$\%$ based on total number of} studies $\mathbf{n}=\mathbf{2 3}$

Captions: "1":Psychotropics could not be classified according to the pharmacological subgroup; “2”:These medications were classified according to ATC Therapeutic Subgroup ( $2^{\text {nd }}$ level $)$. 
1996; Vassalo et al., 2006; Aisen, Deluca, Lawlor, 1992). Four studies consisted of only elderly patients while five others included special population. The medication in this subgroup included all benzodiazepines. Ten studies did not specify the chemical substance (eg. Benzodiazepines NE or Anxiolytics NE). The reports mentioned the following medications: alprazolam, bromazepam, chlordiazepoxide, clobazam, clonazepam, clorazepate, clotiazepam, cloxazolam, diazepam, ethyl loflazepate, etizolam, lorazepam, midazolam, nitrazepam, oxazepam, temazepam and triazolam.

\section{Hypnotics and sedatives}

Data on the use of hypnotics and sedatives were collected from 11 studies. Among these studies, two study populations were, however, restricted to elderly patients. Most of the studies did not specify the medication employed in their investigation (Hayakawa et al., 2014; Kolla et al., 2013; Costa-Dias et al., 2014; Tanaka et al., 2008; Chang et al., 2011; Krauss et al., 2005) while four others mentioned the following medications: zolpidem, zopiclone (Kolla et al., 2013; Dauphinot et al., 2014; Chang et al., 2011), brotizolam, flunitrazepam, lormetazepam, midazolam, nitrazepam, quazepam, rilmazafone, triazolam (Shuto et al., 2010; Obayashi et al., 2013; Mendelson, 1996).

\section{Antipsychotics}

Eight studies attributed inpatient falls to antipsychotics. Most of the studies were devoted to special populations (Tapper et al., 2015; Costa-Dias et al., 2014; Vassalo et al., 2006; Aisen, Deluca, Lawlor, 1992; Bun, Serby, Friedmann, 2011) and only three placed no restrictions on the study population (Lamis et al., 2012; Dauphinot et al., 2014; Mendelson, 1996). The chemical substances included: olanzapine, risperidone, amisulpride, tiaprida, ciamemazine, quetiapine and haloperidol. Four studies did not specify the medication involved.

\section{Opioids}

Seven studies analyzed the use of opioids and the incidence of accidental falls (Walker, 2005; Pierce et al., 2013, Tanaka et al., 2008). Four of these studies contained special populations (Mamun, Lim, 2009; Chang et al., 2011; Dauphinot et al., 2014; Costa-Dias et al., 2014). Tramadol was the only substance specified in a single study. The other studies mentioned opioids, yet without specifying them.

\section{Antiepileptics}

Data on the use of antiepileptics were collected from seven studies (O'Neil et al., 2015; Lamis et al., 2012; Dauphinot et al., 2014; Walker, 2005; Obayashi et al., 2013). Two of these studies focused their investigation on elderly patients and patients with chronic kidney disease (Costa-Dias et al., 2014; Angalakuditi, Gomes, Coley, 2007). The chemical substances cited were carbamazepine, gabapentin, lamotrigine, oxcarbazepine, phenytoin, topiramate, valproate and phenobarbital. Four studies did not specify the drugs employed in their investigation.

\section{Antidepressants}

Five studies associated inpatient falls with antidepressants (O’Neil et al., 2015; Lamis et al., 2012; Mendelson, 1996), two of which focused their investigation on elderly patients and patients with chronic kidney disease (Costa-Dias et al., 2014; Angalakuditi, Gomes, Coley, 2007). The chemical substances cited included amitriptyline, fluoxetine, nortriptyline, clomipramine, doxepin, desipramine, phenylpiperazine, imipramine and sertraline.

\section{DISCUSSION}

Our critical review shows that the use of central nervous system drugs (including anxiolytics; hypnotics and sedatives; antipsychotics; opioids; antiepileptics and antidepressants) is likely to induce inpatient fall incidents.

To the best of our knowledge, there are no reports in the literature concerning the analysis of particular medication related to fall incidents involving hospitalized patients. The studies that have been published recently were aimed at different patient settings such as community and long-term facilities. Interestingly, the findings of these studies were quite similar to ours. Wolcott (2009) conducted a systematic search and a meta-analysis aiming at updating the study carried out by Leipzig, Cumming and Tinetti (1999). They evaluated all FRIDS (Fall Risk Increasing Medication) in different settings (community, long-term facilities and hospital care). The investigation included 22 observational studies and the outcome was based on the Bayesian random effects-model. The result of their investigation pointed out an association between the elderly who use sedatives/ hypnotics, antidepressants and benzodiazepines and accidental falls. Park et al. (2015) reported the results of a systematic investigation they carried out which associated the use of sedatives/hypnotics 
and antidepressants with an increased risk of fall among the elderly. The critical review of Hartikainen, Lonnroos and Louhivuori (2007) associated central nervous system medications with fall incidents among older people. The researcher also suggested some topics that could help improve the quality of observational studies employed towards examining medication use as a risk factor for falling. All the above-mentioned researchers have pointed out the underlying limitations of their studies with regard to quality, consistency, and comparability. They acknowledged the need for well-designed studies such as those related to prospective cohorts. It is worth noting that our study encountered similar limitations.

A famous tool recommended by the Agency of Health Research and Quality (AHRQ, 2016) which is based on the study conducted by Beasley and Patatanian (2009) classified drugs according to the risk of falling for patients in hospital settings. This tool evaluates medication-related fall risk on admission based on a score. Analgesics, antipsychotics, anticonvulsants, benzodiazepines were assigned 3 points which denoted high risk of fall; antihypertensives, cardiac drugs, antiarrhythmics and antidepressants scored 2 points (denoting medium risk); and diuretics gained one point (indicating low risk). In our study, the association between inpatient fall and other pharmacological subgroups (unlike the central nervous system drugs) was found to be weak (only less than $20 \%$ of the studies showed this relation). Some studies published in the literature included antihypertensives, cardiac drugs, antiarrhythmics and diuretics as having weak risk of causing inpatient falls (Leipizig, Cumming, Tinetti, 1999).

Evidence from healthcare settings is supported by the prominent tool known as the evidence pyramid (Murad et al., 2016). The case control design, represented by $61 \%$ of the total eligible studies, is known to have low quality of evidence (it is found at the base of the evidence pyramid). The starting point of this study is the outcome (which in our case is patient falls in hospital settings). Afterwards, an assessment is made regarding the exposure to the risk so as to develop a hypothesis with respect to risk estimation. It is true that this risk estimation does not often reflect the real causality (Nobre, Bernardo, 2006). Moreover, in many studies the control group selection appears to be inadequate and fails to represent the population in an appropriate fashion (Figure 2). No randomized controlled trial (RCT) experiments are found in our investigation. This is attributed to the fact that this type of study design has not been used routinely for the evaluation of adverse effects. The article that reports the new evidence pyramid highlights the critical assessment of the studies taking into account methodological limitations, imprecision, inconsistency and indirectness. These topics could be analyzed through the tool for assessing risk of bias (Figure 2). Only 8 studies were found to have low risk of bias in three or more items that were considered critical to the quality of the study.

Retrospective studies accounted for $87 \%(n=20)$ of the total eligible studies. Several limitations were noted regarding this type of study. These included the inappropriate nature of chart review for study questions or lack of systematic review, the unavailability of important data, the difficulty of establishing cause and effect, the reliability of written information and the problem of missing data (Hess, 2004).

Some studies presented several problems with respect to statistical analysis. In one of the studies, the statistical analysis was not homogeneous for the groups under investigation (some had odds ratio (OR), whereas others did not). Some studies did not present confidence interval (CI) despite having defined an OR value. In many studies, CI was close to 1 (1.01 to 1.09) and the results that were equal or above 1 show no relationship between cause and effect (Bland, 2000). In one study employing crosssectional design, the statistical analysis was not performed at all. The analysis was restricted to medications that were related to fall incidents which the author found in the literature. As no control group was assessed, causality could also not be evaluated.

One of the study limitations stemmed from the groupings of medication. Some studies grouped different pharmacological subgroups under the same category. For purposes of illustration, the studies employed categories such as "medication that can produce sedation or postural hypotension", "central nervous system medication" or "psychotropic medication". Clearly, this was a relevant weakness inasmuch as the full analysis was performed relative to a broad group, and could not, as a result, be strictly attributed to a specific pharmacological group or medication.

Limitations were likewise observed in most of the studies regarding the evaluation of confounders. The bias analysis tool (Figure 2) showed that only 8 studies had a relatively satisfactory identification. It is a known fact that fall incidents are attributed to multifactorial reasons (Spoelstra, Given, Given, 2012), as such, a complete list of potential confounders is essentially important if one is to appropriately evaluate bias that may exclude the relation between a given medication and inpatient fall.

The external validity of the studies faces constraints in that $91 \%(n=21)$ of them were performed in a single institution. A further shortcoming lies in the fact that a significant number of studies focused their investigation 
on specific populations including cirrhotic patients, psychiatric patients, patients suffering from chronic kidney disease, confused patients, elderly patients, patients with altered gait and those suffering from geropsychiatry. The inclusion criteria for one of the studies were restricted to patients exposed to anticholinergic and sedative medications. One will notice that these conditions by themselves could be related to fall incidents (Soriano et al., 2012; Ruxton, Woodman, Mangoni, 2015; Severo et al., 2014; Spoelstra, Given, Given, 2012).

The median age of the fallers was 69 years; this can largely be explained by the fact that elderly patients are more prone to accidental falls and age itself is cited as a single risk factor (WHO, 2007). In Brazil, 33.5\% of patients in the Brazilian Public Health Service (known as Sistema Único de Saúde - SUS) are aged 60 and above (Peixoto et al., 2004). The US reported similar percentages of elderly inpatients in 2012 , where $34.9 \%$ of all hospitalizations represented people aged 65 and above. Indeed, this shows that the elderly population accounts for a significant amount of hospital admissions.

One of our study constraints was related to the use of the quality assessment tool adapted from STROBE and Young and Solomns (2009) instead of the well-known observational assessment tool (like Newcastle-Ottawa Scale). We chose this adapted tool owing to the fact that it provides the possibility of locating all eligible studies in a single table and irrespective of study design. In addition, it gives a clearer exposition of information with self-explanatory icons (the caption was inspired by the Cochrane collaboration tool for assessing risk of bias in randomized trials).

Other study constraints our study encountered were related to ATC classification which could not be fitted to all studies and drugs, considering the indication of some specific medications or group classification. Grey literature was not included and our search was restricted to English, Spanish and Portuguese languages. The review steps were performed by only one of the authors.

The assessment of fall risk in hospital settings is extremely important as it can help prevent inpatient negative outcomes and contribute towards increasing the safety policy of the institution (WHO, 2008). Notwithstanding the weakness of some studies included in this review, our findings were similar to those of the most recent reports published in the literature which also associated central nervous system drugs (namely anxiolytics; hypnotics and sedatives; antipsychotics; opioids; antiepileptics and antidepressants) with inpatient falls. These findings could be used as a tool to help prevent inpatient falls, especially for those patients who exhibit more risk factors for fall incidents. The multi-professional team acting in inpatient healthcare settings can contribute towards improving patient safety and help diminish fall incidents. One of the mechanisms to prevent patient falls in hospitals is assigning a professional pharmacist to the team and allowing him/her to review the medical records and prescriptions of the patients (Zermansky et al., 2006; Haumschild et al., 2003). Similarly, the hospital nursing team can tailor their care planning activities in such a way as to prevent negative outcomes (including inpatient falls) during the stay of the patients in healthcare settings (Severo et al., 2014; Hignett, Masud, 2006).

\section{CONCLUSION}

Our study shows that central nervous system medications, particularly in the following order: anxiolytics, hypnotics and sedatives, antipsychotics, opioids, antiepileptics and antidepressants, seem to be associated with an increased risk of inpatient falling. The result of this study is in line with those of recently published reports in the literature on risk of fall incidents in community and other settings. While the results of this critical review are worthy of consideration, one cannot virtually neglect the several methodological limitations that characterize the eligible observational studies that were employed. Among the constraints noted in these eligible studies included failure to provide proper definition, unspecified medication classifications and unsatisfactory description of potential confounders. Better delineated studies are needed if one is to properly assess the relationship between medications and inpatient falls.

\section{ACKNOWLEDGEMENT}

The authors would like to express their sincerest gratitude and indebtedness to both the Brazilian ministries of health and education for the financial support provided during the course of this study and for the authors' clinical pharmacy residency program.

\section{REFERENCES}

Abreu H, Reiners A, Azevedo R, Silva A, Abreu D, Oliveira A. Incidence and predicting factors of falls of older inpatients. Rev Saúde Públ. 2015;49:13-20.

Agency for Healthcare Research and Quality. AHRQ. Tool 3I: Medication fall risk score and evaluation tools. [cited on: 16 Nov. 2016]. Available from: http://www.ahrq.gov/professionals/ systems/hospital/fallpxtoolkit/fallpxtk-tool3i.html. 
Aisen P, Deluca T, Lawlor B. Falls among geropsychiatry inpatients are associated with prn medications for agitation. Int J Geriat Psych. 1992;7(10):709-712.

Angalakuditi M, Gomes J, Coley K. Impact of drug use and comorbidities on in-hospital falls in patients with chronic kidney disease. Ann Pharmacother. 2007;41(10):1638-1643.

Beasley B, Patatanian E. Development and implementation of a pharmacy fall prevention program. Hosp Pharm. 2009;44(12):1095-1102.

Bland J. Statistics notes: the odds ratio. BMJ. 2000;320(7247):1468-1468.

Bloch F, Thibaud M, Dugue B, Breque C, Rigaud A, Kemoun G. Psychotropic drugs and falls in the elderly people: updated literature review and meta-analysis. J Aging Health. 2010;23(2):329-346.

Bouldin E, Andresen E, Dunton N, Simon M, Waters T, Liu M, et al. Falls among adult patients hospitalized in the United States. J Patient Safety. 2012;9(1)13-17.

Boushon B, Nielsen G, Quigley P, Rutherford P, Taylor J, Shannon D, Rita S. How-to guide: reducing patient injuries from falls. Cambridge, MA: Institute for Healthcare Improvement; 2012. Available from: www.ihi.org.

Boyle N, Naganathan V, Cumming R. Medication and falls: risk and optimization. Clin Geriat Med. 2010;26(4):583-605.

Brasil. Ministério da Saúde. Fall prevention protocol. Protocolo de prevenção de quedas. 2013b. [cited 4 Jan. 2017]. Available from: http://www20.anvisa.gov.br/segurancadopaciente/index. $\mathrm{php} /$ publicacoes/item/prevencao-de-quedas.

Browne C, Kingston C, Keane C. Falls prevention focused medication review by a pharmacist in an acute hospital: implications for future practice. Int J Clin Pharm. 2014;36(5):969-975.

Buksman S, Vilela ALS, Pereira SEM, Lino VS, Santos VH. Projeto Diretrizes. Quedas em idosos: prevenção. 2008. [cited 16 Nov. 2016]. Available from: http://sbgg.org.br/wp-content/ uploads/2014/10/queda-idosos.pdf.

Bun S, Serby M, Friedmann P. Psychotropic medication use, hyponatremia, and falls in an inpatient population. J Clin Psychopharmacol. 2011;31(3):395-397.
Campbell A. Drug treatment as a cause of falls in old age. Drugs Aging. 1991;1(4):289-302.

Cashin R, Yang M. Medications prescribed and occurrence of falls in general medicine inpatients. Can J Hosp Pharm. 2011;64(5):321-326.

Centers for Disease Control and Prevention. CDC. 2014. [cited on: 16 Nov. 2016]. Available from: https://www.cdc.gov/nchs/ index.htm.

Chang C, Chen M, Tsai C, Ho L, Hsieh H, Chau Y, et al. Medical conditions and medications as risk factors of falls in inpatient older people: a case-control study. Int J Geriat Psych. 2011;26(6):602-607.

Costa-Dias M, Oliveira A, Martins T, Araújo F, Santos A, Moreira $\mathrm{C}$ et al. Medication fall risk in old hospitalized patients: A retrospective study. Nurse Edu Today. 2014;34(2):171-176.

DataSUS. Departamento de Informática do SUS. DataSUS. 2014. [cited: 16 Nov. 2016]. Available from: http://www2. datasus.gov.br/DATASUS/index.php?area=02.

Dauphinot V, Faure R, Omrani S, Goutelle S, Bourguignon L, Krolak-Salmon P, et al. Exposure to anticholinergic and sedative drugs, risk of falls, and mortality. J Clin Psychopharmacol. 2014;34(5):565-570.

Ferreira D, Yoshitome A. Prevalência e caraterísticas das quedas de idosos institucionalizados. Rev Bras Enferm. 2010;63(6):991-997.

Frels C. Iatrogenic causes of falls in hospitalised elderly patients: a case-control study. Postgrad Med J. 2002;78(922):487-489.

Hartikainen S, Lonnroos E, Louhivuori K. Medication as a risk factor for falls: critical systematic review. J Gerontol Ser A: Biol Sci Med Sci. 2007;62(10):1172-1181.

Haumschild MJ, Karfonta TL, Haumschild MS, Phillips SE. Clinical and economic outcomes of a fall-focused pharmaceutical intervention program. Am J Health-Syst Pharm. 2003;60(10):1029-1032.

Hayakawa T, Hashimoto S, Kanda H, Hirano N, Kurihara Y, Kawashima T, et al. Risk factors of falls in inpatients and their practical use in identifying high-risk persons at admission: Fukushima Medical University Hospital cohort study. BMJ Open. 2014;4(8):e005385-e005385. 
Hegeman J, van den Bemt B, Duysens J, van Limbeek J. NSAIDs and the risk of accidental falls in the elderly. Drug Safety. 2009;32(6):489-498.

Hess DR. Retrospective studies and chart reviews. Respir Care 2004;49(10):1171-4.

Hignett S, Masud T. A review of environmental hazards associated with in-patient falls. Ergonomics. 2006;49(5-6):605616.

Kolla B, Lovely J, Mansukhani M, Morgenthaler T. Zolpidem is independently associated with increased risk of inpatient falls. J Hosp Med. 2013;8(1):1-6.

Krauss M, Evanoff B, Hitcho E, Ngugi K, Dunagan W, Fischer I, et al. A case-control study of patient, medication, and carerelated risk factors for inpatient falls. J Gen Internal Med. 2005;20(2):116-122.

Lamis R, Kramer J, Hale L, Zackula R, Berg G. Fall risk associated with inpatient medications. Am J Health-Syst Pharm. 2012;69(21):1888-1894.

Leipzig R, Cumming R, Tinetti M. Drugs and falls in older people: a systematic review and meta-analysis: i. psychotropic drugs. J Am Geriatr Soc. 1999;47(1):30-39.

Mamun K, Lim J. Association between falls and high-risk medication use in hospitalized Asian elderly patients. Geriatr Gerontol Int. 2009;9(3):276-281.

Matarese M, Ivziku D, Bartolozzi F, Piredda M, De Marinis M. Systematic review of fall risk screening tools for older patients in acute hospitals. J Adv Nursing. 2014;71(6):1198-1209.

Mendelson WB. The use of sedative/hypnotic medication and its correlation with falling down in the hospital. Sleep. 1996;19(9):968-701.

Miake-Lye I, Hempel S, Ganz D, Shekelle P. Inpatient fall prevention programs as a patient safety strategy. Ann Internal Med. 2013;158(5 Part 2):390.

Murad M, Asi N, Alsawas M, Alahdab F. New evidence pyramid. Evid Based Med. 2016;21(4):125-127.

Nobre MRC, Bernardo WM. Prática clínica em evidência. São Paulo: Elsevier; 2006.
O’Neil C, Krauss M, Bettale J, Kessels A, Costantinou E, Dunagan W, et al. Medications and patient characteristics associated with falling in the hospital. J Patient Safety. 2015;1.

Obayashi K, Araki T, Nakamura K, Kurabayashi M, Nojima Y, Hara K, et al. Risk of falling and hypnotic drugs: retrospective study of inpatients. Drugs in R D. 2013;13(2):159-164.

Park H, Satoh H, Miki A, Urushihara H, Sawada Y. Medications associated with falls in older people: systematic review of publications from a recent 5-year period. Eur J Clin Pharmacol. 2015;71(12):1429-1440.

Peixoto SV, Giatti L, Afradique ME, Lima-Costa, F. Custo das internações hospitalares entre idosos brasileiros no âmbito do Sistema Único de Saúde. Epidemiol Serv Saúde. 2004;13(4):239-246.

Pierce JR, Shirley M, Johnson EF, Kang H. Narcotic administration and fall-related injury in the hospital: Implications for patient safety programs and providers. Int J Risk Safety Med. 2013;25(4):229-234.

Rezende CP, Gaede-Carrillo MRG, Sebastião ECO. Queda entre idosos no Brasil e sua relação com o uso de medicamentos: revisão sistemática. Cad Saúde Pub. 2012;28(12):2223-2229.

Richardson K, Bennett K, Kenny R. Polypharmacy including falls risk-increasing medications and subsequent falls in community-dwelling middle-aged and older adults. Age Ageing. 2014;44(1):90-96.

Rubenstein L. Falls in older people: epidemiology, risk factors and strategies for prevention. Age Ageing. 2006;35:(Suppl 2):ii37-ii41.

Ruxton K, Woodman R, Mangoni A. Drugs with anticholinergic effects and cognitive impairment, falls and all-cause mortality in older adults: A systematic review and meta-analysis. Brit J Clin Pharmacol. 2015;80(2):209-220.

Severo I, Almeida M, Kuchenbecker R, Vieira D, Weschenfelder M, Pinto L, et al. Risk factors for falls in hospitalized adult patients: an integrative review. Rev Escola Enferm USP. 2014;48(3):540-554.

Shuto H, Imakyure O, Matsumoto J, Egawa T, Jiang Y, Hirakawa $\mathrm{M}$, et al. Medication use as a risk factor for inpatient falls in an acute care hospital: a case-crossover study. Brit J Clin Pharmacol. 2010;69(5):535-542. 
Soriano G, Román E, Córdoba J, Torrens M, Poca M, Torras $\mathrm{X}$, et al. Cognitive dysfunction in cirrhosis is associated with falls: A prospective study. Hepatology. 2012;55(6):1922-1930.

Soriano G, Román E, Córdoba J, Torrens M, Poca M, Torras $\mathrm{X}$, et al. Cognitive dysfunction in cirrhosis is associated with falls: A prospective study. Hepatology. 2012;55(6):1922-1930.

Spoelstra S, Given B, Given C. Fall prevention in hospitals: an integrative review. Clin Nursing Res. 2012;21(1):92-112.

Tanaka M, Suemaru K, Ikegawa Y,Tabuchini N, Araki H. Relationship between the risk of falling and drugs in an academic hospital. Yakugaku Zasshi. 2008;128(9):1355-1361.

Tapper E, Risech-Neyman Y, Sengupta N. Psychoactive medications increase the risk of falls and fall-related injuries in hospitalized patients with cirrhosis. Clin Gastroenterol Hepatol. 2015;13(9):1670-1675.

Vassallo M, Vignaraja R, Sharma J, Briggs R, Allen S. Predictors for falls among hospital inpatients with impaired mobility. JRSM. 2004;97(6):266-269.

Vassallo M, Vignaraja R, Sharma J, Briggs R, Allen S. Tranquiliser use as a risk factor for falls in hospital patients. Int J Clin Pract. 2006;60(5):549-552.

von Elm E, Altman DG, Egger M, Pocock SJ, Gøtzsche PC, Vandenbroucke JP, STROBE Initiative. Strengthening the reporting of observational studies in epidemiology (STROBE) statement: guidelines for reporting observational studies. BMJ. 2007;335(7626):806-8.

Walker P. Medication use as a risk factor for falls among hospitalized elderly patients. Am J Health-Syst Pharm. 2005;62(23):2495-2499.
Wong C, Recktenwald A, Jones M, Waterman B, Bollini M, Dunagan W. The cost of serious fall-related injuries at three Midwestern Hospitals. Joint Comm J Quality Patient Safety. 2011;37(2):81-87.

Woolcott J. Meta-analysis of the impact of 9 medication classes on falls in elderly persons. Arch Internal Med. 2009;169(21):1952.

World Health Organization. WHO. Global report on falls prevention in older age WHO global report on falls prevention in older age. Geneva: WHO; 2007. p.1-7.

World Health Organization. WHO. The global burden diseases. 2004. [cited: 16 Nov. 2016]. Available from: http://www.who. int/healthinfo/global_burden_disease/2004_report_update/en/

World Heathy Organization. WHO. World Alliance for Patient Safety. Summary of the evidence on patient safety: implications for research. Geneva: WHO; 2008. 136 p.

Young J, Solomon M. How to critically appraise an article. Nat Clin Pract Gastroenterol Hepatol. 2009;6(2):82-91.

Zang G. Antihypertensive drugs and the risk of fall injuries: A systematic review and meta-analysis. J Int Med Res. 2013;41(5):1408-1417.

Zermansky A, Alldred D, Petty D, Raynor D, Freemantle N, Eastaugh J, et al. Clinical medication review by a pharmacist of elderly people living in care homes--randomised controlled trial. Age Ageing. 2006;35(6):586-591.

Received for publication on $14^{\text {th }}$ June 2017 Accepted for publication on $27^{\text {th }}$ October 2017 